\title{
Arborescences
}

Revue d'études françaises

\section{Ce que la lettre familière fait au discours médical. Une lecture de la lettre XIX, 16 des Lettres de Pasquier (1619)}

\section{Benoît Autiquet}

Numéro 9, décembre 2019

La lettre érudite. Nouvelles recherches sur la communication savante à l'époque moderne (XVI ${ }^{\mathrm{e}}$-XVIII ${ }^{\mathrm{e}}$ siècles)

URI : https://id.erudit.org/iderudit/1068273ar

DOI : https://doi.org/10.7202/1068273ar

Aller au sommaire du numéro

Éditeur(s)

Département d'études françaises, Université de Toronto

ISSN

1925-5357 (numérique)

Découvrir la revue

Citer cet article

Autiquet, B. (2019). Ce que la lettre familière fait au discours médical.

Une lecture de la lettre XIX, 16 des Lettres de Pasquier (1619). Arborescences,

(9), 31-48. https://doi.org/10.7202/1068273ar
Résumé de l'article

L'article porte sur la lettre XIX, 16 du second tome des Lettres de Pasquier, publié en 1619. Cette lettre, qui traite de la science médicale de l'époque de l'auteur, est généralement lue comme un lieu où Pasquier, refusant les dogmes médicaux au nom du scepticisme, fait valoir la vérité de l'expérience personnelle en matière médicale. Nous essayons de montrer au contraire que, loin de valoriser uniquement le savoir individuel, cette lettre, très critique envers les dogmes médicaux, propose néanmoins des formes institutionnelles du savoir médical. Mais celles-ci, contrairement aux « écoles " antiques, ne sont pas construites autour d'un centre dogmatique. Deux modèles institutionnels, concurrents et parfois contradictoires, sont proposés par l'auteur : un modèle divin, qui fait des médecins des agents de Dieu ; un modèle humain, où les médecins ont le rôle de « compilateurs " des remèdes locaux qui, conformément à la théorie médicale des climats, sont les seuls à pouvoir soigner les habitants d'un lieu. On propose, en fin de parcours, une tentative pour articuler ces deux modèles.
Tous droits réservés () Département d'études françaises, Université de Toronto, 2020
Cedocument est protégé par la loi sur le droit d'auteur. L'utilisation des services d'Érudit (y compris la reproduction) est assujettie à sa politique d'utilisation que vous pouvez consulter en ligne.

https://apropos.erudit.org/fr/usagers/politique-dutilisation/ 


\section{SOMMAIRE}

1 Sébastien Drouin, Université de Toronto

Camelia Sararu, Université de Toronto

Introduction

10 Myron McShane, Centre d'études sur la Réforme et la Renaissance, Université de Toronto Une lettre de Jean Dorat sur l'œuvre de Nonnos

31 Benoît Autiquet, Université de Bâle

Ce que la lettre familière fait au discours médical.

Une lecture de la lettre XIX, 16 des Lettres de Pasquier (1619)

49 Guillaume Bazière, Université Paris Nanterre

Présence érudite et savoirs politiques dans la correspondance du Grand Condé

68 Vanezia Pârlea, Université de Bucarest

Lettres d'Orient : échanges épistolaires en contexte interculturel dans les Mémoires du chevalier d'Arvieux

81 Yves Moreau, Université de Lyon

"Qualche novità litteraria»: la correspondance

entre Jacob Spon (1647-1685) et Antonio Magliabechi (1633-1714)

95 Corinne Marchal, Université de Franche-Comté

La dynamique de la circulation des savoirs et de leurs matériaux dans les échanges épistolaires entre Jean-Baptiste Boisot et Paul Pellisson-Fontanier (1674-1693)

106 Mathilde Chollet, Le Mans Université

Un «gai savoir»: stratégies du rire dans les lettres

$d$ 'une érudite des Lumières 


\title{
Ce que la lettre familière fait au discours médical. Une lecture de la lettre XIX, 16 des Lettres de Pasquier (1619)
}

\author{
Benoît Autiquet, Université de Bâle
}

\section{Résumé}

L'article porte sur la lettre XIX, 16 du second tome des Lettres de Pasquier, publié en 1619. Cette lettre, qui traite de la science médicale de l'époque de l'auteur, est généralement lue comme un lieu où Pasquier, refusant les dogmes médicaux au nom du scepticisme, fait valoir la vérité de l'expérience personnelle en matière médicale. Nous essayons de montrer au contraire que, loin de valoriser uniquement le savoir individuel, cette lettre, très critique envers les dogmes médicaux, propose néanmoins des formes institutionnelles du savoir médical. Mais celles-ci, contrairement aux "écoles» antiques, ne sont pas construites autour d'un centre dogmatique. Deux modèles institutionnels, concurrents et parfois contradictoires, sont proposés par l'auteur: un modèle divin, qui fait des médecins des agents de Dieu; un modèle humain, où les médecins ont le rôle de "compilateurs » des remèdes locaux qui, conformément à la théorie médicale des climats, sont les seuls à pouvoir soigner les habitants d'un lieu. On propose, en fin de parcours, une tentative pour articuler ces deux modèles.

Dans son récent ouvrage Extraordinaire et ordinaire des Croix. Les représentations du corps souffrant (1580-1650), Antoinette Gimaret a proposé une lecture du discours médical dans les Lettres de Pasquier. Elle situe l'émergence de ce discours au croisement de deux phénomènes intellectuels et culturels. Le premier est l'apparition au cours $\mathrm{du} \mathrm{XvI}^{\mathrm{e}}$ siècle d'une épistémologie médicale qui explore les «singularités», ou, pour reprendre le titre d'une sous-partie de son ouvrage, fait surtout "attention au particulier» (Gimaret 2011: 653). Le malade est avant tout considéré comme un «cas» dont le médecin, proche en cela du directeur de conscience, doit attentivement observer les particularités pour proposer une cure efficace. Le second phénomène intellectuel est le développement des "écritures du for privé», lui-même conséquence d'une «émergence de l'individu " dont les «[1] ettres familières, mémoires, essais, journaux» (Gimaret 2011: 677) qui fleurissent à la fin de la Renaissance seraient le marqueur. Plus particulièrement, les «lettres familières» portent en elles une nouvelle manière d'écrire le savoir, car, se substituant à la "rhétorique savante, latiniste, érudite» et «trouv[a]nt dans la lettre familière de type cicéronien un modèle adéquat», elles peuvent prendre en charge des «anecdotes quotidiennes» et «une mise en discours de l'intime» fait par "un "je" singulier qui parle en son nom propre»(Gimaret 2011 : 700-701). Commentant, à propos des Lettres de Pasquier, cette coïncidence entre un genre littéraire et une tendance épistémologique, Antoinette Gimaret écrit :

Comme il est, selon l'épistolier, indispensable au médecin de "philosopher sur la façon de son malade", c'est-à-dire sur la variété des saisons, des lieux et des âges, mais aussi des mœurs, des nourritures et de la force de chacun des individus qu'il examine, il est essentiel à Pasquier de se raconter particulièrement, dans la santé et dans la maladie. (Gimaret 2011:717) 
Ainsi la tendance de l'auteur à se raconter dans certains genres entre en résonance avec une épistémologie médicale qui fait de la prise en compte du patient en tant qu'individu, considéré dans un contexte particulier, une condition essentielle de l'efficacité thérapeutique. La lettre familière, dans sa capacité de dire le «moi» intime, est le lieu idéal pour mener la critique d'une médecine qui «a le défaut d'être une science dogmatique, trop éloignée parfois des expériences singulières» (Gimaret 2010: n. p p).

L'idée que la lettre familière est le lieu d'une émancipation de l'individu par rapport à des carcans sociétaux et dogmatiques n'est pas nouvelle. Elle a ses racines dans l'article fondateur de Marc Fumaroli «Genèse de l'épistolographie classique: rhétorique humaniste de la lettre, de Pétrarque à Juste Lipse», qui voit dans la naissance de l'épistolarité familière le lieu d'une émergence stylistique de l'individu. La Renaissance hérite des forme dictandi et des artes dictaminis médiévaux qui ont "gelé pour ainsi dire le genre dans une pratique administrative de secrétaires" (Fumaroli 1978: 887). Cette pratique épistolaire, qui s'avère "incapable de transcrire le frémissement intérieur de l'âme chrétienne», est révolutionnée par Pétrarque chez qui «c'est la personne intime et privée, et non une persona officielle, qui est l'auteur de la lettre, même lorsqu'elle s'adresse à un grand personnage». Ainsi, l'épistolarité pétrarquiste propose une " "peinture" du moi intime de l'épistolier» (Fumaroli 1978: 888), que reprennent les lettres familières du Xvi siècle, via Érasme et Juste Lipse. Luc Vaillancourt, dans le seul ouvrage entièrement consacré à l'épistolarité familière de langue française au XvI ${ }^{\mathrm{e}}$ siècle, insiste lui aussi sur l'idée d'une émancipation de l'individualité auctoriale. Analysant les Lettres de Pasquier, il écrit: "Pasquier entreprend de peindre les méandres d'un moi sur lequel pèsent des tabous séculaires avec une pudeur qui s'évanouit au fil du temps et une cohérence qui ne cesse de croître» (Vaillancourt 2003: 391-392). Le recueil épistolaire est donc à la fois le point d'aboutissement d'une lente émancipation historique de l'individu, et la trace du processus par lequel celui-ci, au fil de sa vie, se libère de la "pudeur» qui le tenait jusque-là enfermé dans des contraintes sociales. La lettre familière serait l'un des genres où, au XVI ${ }^{\mathrm{e}}$ siècle, se joue "la capacité des membres d'une société à s'émanciper de la doxa et de son totalitarisme idéologique» (Vaillancourt 2003: 396). Malgré la diversité des propositions d'Antoinette Gimaret, de Luc Vaillancourt et de Marc Fumaroli ${ }^{2}$, on ne peut qu'être frappé par leur conception commune de l'écriture épistolaire comme dynamique d'émancipation de l'individu par rapport à des carcans formels ou sociétaux qui pèseraient sur lui. Gimaret hérite donc de cette tradition critique, et, à la libération individuelle que permettrait l'écriture épistolaire, elle fait correspondre la libération épistémologique qu'offrirait l'exploration du cas par rapport au dogme médical.

Or, la lecture de la principale lettre du recueil consacrée à la médecine (XIX, 16), adressée à «Monsieur Tournebus», c'est-à-dire à l'un des fils d'Adrien Turnèbe, Odet ou Nicolas ${ }^{3}$, invite à modifier cette conception du rapport entre savoir individuel et savoir dogmatique dans le discours médical des Lettres de Pasquier. En effet, s'il insiste effectivement sur le savoir expérientiel et individuel,

1. Nous citons ici un article d'Antoinette Gimaret exclusivement consacré à Pasquier, où elle reprend l'essentiel des analyses proposées dans son ouvrage (Gimaret 2010).

2. On pense, notamment, au rôle de la religion chrétienne, que Marc Fumaroli présente comme le ferment d'une émancipation de l'individu, et qui ne possède pas cette fonction chez Luc Vaillancourt et Antoinette Gimaret.

3. Sur la relation entre Étienne Pasquier et les Turnèbe, et l'inscription de cette relation dans le recueil des Lettres, voir Magnien 1999: 268-292. Sur la difficulté d'identifier lequel des deux fils Turnèbe (Odet ou Nicolas) est le véritable destinataire, voir la discussion des positions de Dorothy Thickett par Catherine Magnien (Magnien 1999: 276 et suiv.) Cette dernière reconnaît cependant que le destinataire semble avoir peu d'impact sur le contenu de la lettre sur la médecine (Magnien 1999: 289-290), qui s'apparente davantage à un discours qu’à une missive adressée à une personne en particulier. C'est pourquoi nous laissons de côté, dans cet article, l'éventuelle influence du destinataire sur les opinions médicales de Pasquier. 
l'épistolier ne l'oppose pas aux dogmes généraux, mais cherche au contraire à les penser ensemble. C'est que, sur la question du rapport à l'institution médicale, Pasquier, comme on le verra, n'est ni Pline, ni Montaigne. Ces derniers, à partir du double constat de l'impuissance des médecins à guérir et de l'efficacité d'une thérapeutique individuelle ou familiale, en déduisent la nécessité de bannir les médecins ou de se garder de leur fréquentation; au contraire, Pasquier maintient un lien personnel avec les médecins et souligne sa volonté de définir l'utilité sociale de leur profession. Cela modifie considérablement le cour problématique de la lettre qui nous intéresse: la question qu'elle soulève est moins celui celle de la mise en valeur d'une expérience personnelle contre un dogme institué, que celle de l'institution d'une discipline dont le dogme central est introuvable. Ainsi, plutôt que de déduire de l'absence de dogme unique l'impossibilité d'une institution médicale efficace, Pasquier préfère réfléchir à la manière d'unir les médecins dans une même institution malgré l'absence de dogme. Or, on verra que cette institution d'une discipline non dogmatique est à ce point problématique que Pasquier en propose deux modèles, qui semblent difficilement conciliables entre eux: un modèle divin et un modèle humain. Évidemment, les définitions d' «institution » qu'impliquent ces deux modèles sont très différentes, et nous les préciserons en leur lieu. Enfin, on essaiera d'envisager une manière de penser l'articulation entre ces deux modèles, en replaçant notamment les hésitations qui entourent, dans notre lettre, l'institution humaine de la médecine, dans le contexte plus large des débats qui agitent, à la fin du Xvi ${ }^{\mathrm{e}}$ siècle, le mouvement de vulgarisation du discours médical.

\section{Divisions du dogme médical et intégration sociale des médecins}

La lettre XIX, 16 consiste en une satire des médecins et de la médecine, qui s'inscrit dans une tradition de textes contra medicos, dont la source antique principale est l'Histoire naturelle de Pline l'Ancien, et qui est notamment représentée à l'époque moderne par les Invectivæ contra medicum (1352) de Pétrarque ou par les chapitres concernant la médecine dans le De incertitudine et vanitate omnium scientiarum et artium (1530) de Cornelius Agrippa ${ }^{4}$. L'espace nous manque pour restituer l'ensemble des sources de la lettre XIX, 16 de Pasquier. Nous nous contenterons donc de comparer l'épistolier à sa source principale, Pline l'Ancien, mais aussi à son contemporain Montaigne qui, lui aussi, propose une violente satire des médecins. Cette comparaison portera sur un point précis: le lien entre la critique de la médecine comme dogme, et l'exclusion des médecins de la société.

La critique de la médecine par Pasquier part du constat qu'il n'y a «rien si certain, que l'incertain en cest art» (Pasquier 1619, II, 5395). Pour montrer cette incertitude, lorsqu'il traite de la manière dont la médecine décrit les "particularitez essentielles» de l'homme, Pasquier souligne que, pour chacune de ces particularités, les avis des médecins sont divisés en "part [ies] ». Il déduit de cette incertitude fondamentale de la médecine une incapacité à guérir: "en ceste perplexité, quelle asseurance pouvons nous avoir des remedes que l'on voudra employer pour celuy qui sera malade en l'une de ces fonctions?» (II, 543). Cet argument n'a rien d'original. Il est repris de l'Histoire naturelle de Pline, dont il faut restituer l'argumentaire contre la médecine dans son ensemble pour voir en quoi il se rapproche et differe de celui de Pasquier.

Au chapitre XXIV, I de son Histoire naturelle, l'auteur latin indique qu'il considère la médecine comme une invention frauduleuse, à but avant tout lucratif:

4. Pour un aperçu rapide de cette tradition et de sa réception à la fin du XvI ${ }^{\mathrm{e}}$ siècle, voir Carlino 2002.

5. Désormais abrégé I pour le premier tome ou II pour le second, suivi du numéro de la page. 
La nature ne nous avait destiné que des remèdes partout tout préparés, faciles à trouver et sans aucune dépense [sine inpendio], tirés des substances qui nous font vivre. Plus tard, la fraude humaine et des inventions lucratives [ingeniorum capturæ officinas invenere istas] ont produit ces officines où l'on promet à chacun la vie pour de l'argent. (Pline l'Ancien 1972:26)

Au début du livre XXIX, ayant entretemps fait le tour de l'immense variété des remèdes naturels auxquels «la nature» pourvoit, il prévient une interrogation du lecteur: pourquoi a-t-on oublié tous ces remèdes? Pline retrace alors l'histoire d'une médecine dont l'unique but aurait été de faire croire qu' elle seule pouvait guérir les patients. La théorie médicale n'est jamais qu'un long discours inutile qui vise à faire gagner de l'argent à ceux qui le tiennent; et la variété de ses courants s'explique comme un phénomène de concurrence commerciale: chacun doit faire croire que son produit, ou son remède, ou sa théorie, est celui ou celle qu'il faut acheter. Chez Pline, cette division a des conséquences tragiques: «De là ces misérables querelles d'opinions au chevet des malades, où nul ne veut céder pour ne pas paraître de l'avis d'un autre; de là cette navrante inscription funéraire: mort de trop de médecins» (Pline l'Ancien 1962: 23). De cette histoire d'un dogme médical divisé, frauduleux et impuissant à guérir, Pline procède à une condamnation radicale de la médecine comme profession, prenant comme modèle Caton:

Alors quoi? Devons-nous croire qu'il [Caton] ait condamné un art si utile? Non pas, certes, puisqu'il indique les remèdes qui lui ont permis, à lui et à sa femme d'atteindre une vieillesse avancée, et ces remèdes sont ceux-là mêmes dont nous nous occupons maintenant. Il déclare en outre qu'il a établi, pour son usage, un formulaire qui lui permette de soigner son fils, ses esclaves et ses amis; recueil que nous utilisons ici en le répartissant par catégories d'emploi. Ce n'est pas la chose elle-même que condamnaient les Anciens, mais la profession, car ils n'admettaient surtout pas que la vie puisse dépendre du paiement d'un salaire. (Pline l'Ancien 1962: 25)

La référence à Caton permet à Pline de distinguer entre deux domaines que sa satire avait confondus jusque-là: celui de l'art médical, plus particulièrement celui des "remèdes", et celui de la profession. Le second concentre toutes les critiques, tandis que le premier est préservé. Mais la thérapeutique que Pline valorise est d'un type particulier. Les remèdes de Caton ne s'adressent qu'à ses proches: à luimême, à sa femme et, via un "formulaire», à son fils, à ses esclaves et à ses amis - toutes personnes incluses dans la famille élargie qu'est la domus latine. Il s'agit donc d'une thérapeutique qui se passe de la profession médicale, et qu'on pourrait qualifier d'autarcique. En faisant le tour des remèdes utilisés par Caton, le texte de Pline l'Ancien entend donc se substituer à la pratique d'une médecine frauduleuse et divisée: plutôt que de faire confiance aux médecins dissimulateurs, il suffit de donner au public la connaissance des simples $^{6}$ " que la nature met à sa disposition.

Montaigne est sans doute moins radical dans sa critique, lui qui ne fait que "pincer" la médecine là où Pline «l'esgorg[e]» (Montaigne 2004: 784). Dans le chapitre II, 37 des Essais, "De la ressemblance des enfans aux peres", il formule lui aussi d'abord le constat d'un dogme médical divisé, et donc impuissant à se constituer comme vérité. Il en déduit que c'est une science dans laquelle "fortune vaut bien mieux que la raison» (Montaigne 2004: 764). La médecine est incertaine et non plus frauduleuse comme chez l'auteur latin, mais, à partir de cette critique épistémique, Montaigne formule une conclusion sociale qui ressemble à celle de Pline. Non seulement ni lui ni ses ancêtres n'ont fréquenté de médecins, mais

6. C'est-à-dire d'un remède médical qui n'est composé que d'une seule plante (du latin simplex: «un»). 
[i]l n'est nation qui n'ait esté plusieurs siecles sans la medecine, et les premiers siecles, c'est à dire les meilleurs et les plus heureux; et du monde la dixiesme partie ne s'en sert pas encores à cette heure; infinies nations ne la cognoissent pas, où l'on vit et plus sainement et plus longuement qu'on ne fait icy; et parmy nous le commun peuple s'en passe heureusement. (Montaigne 2004: 766-767 ${ }^{7}$ )

Après quoi Montaigne cite l'exemple de "Caton le Censeur», qui, sans médecin, vécut «quatre vingts et cinq ans, et f[it] vivre sa femme jusqu'à l'extreme vieillesse». On en déduit que la société peut entièrement se passer de médecins. Certes, comme le fait remarquer Dominique Brancher, Montaigne, en bon sceptique, en appelle à des «fantaisies contraires» à la fin du chapitre II, 37 (Brancher 2007 : 743), puisque son exclusion des médecins n'est qu'une "opinion» qui, à ce titre, peut être contestée. Il n'en demeure pas moins qu'à la lecture de cet essai - et en dehors de cet appel à la contradiction qui ressemble à une concession faite à la dédicataire du chapitre, Madame de Duras, amatrice de science médicale -, on se demande quelle pourrait être, aux yeux de Montaigne, la fonction sociale de la profession médicale.

Or, la position de Pasquier sur ce sujet est bien différente. À titre personnel d'abord, sa défiance envers les médecins ne va pas jusqu’à éviter leur fréquentation. Dans la lettre IX, 12, adressée à «Monsieur Seve Docteur en Medecine demeurant à Melun» (ville où réside aussi Pasquier), l'épistolier, à l'occasion d'un «flux de ventre fort aigu», décrit au professionnel les particularités de sa maladie et de sa "complexion", de manière à recevoir de lui "quelque ordonnance». À la fin de cette lettre, il met explicitement à distance le modèle autarcique qui exclurait les médecins :

Ie me fusse volontiers de moy-mesme ordonné une reubarbe, que nous apprenons dans vos livres, avoir une vertu restraignante, $\&$ neantmoins expulsive des malignes humeurs: mais tout ainsi que nos loix civiles nous prohibent d'estre Iuges \& parties en nos causes: aussi les vostres de Medecine defendent de n'estre le Medecin \& le malade tout ensemble. (I, 571)

Le parallèle n'est pas fortuit: Pasquier est avocat et, à ce titre, très attaché au fonctionnement institutionnel de la justice. En posant comme équivalents le "Medecin» et le "Juge», il affirme son attachement à la profession médicale et son refus d'une société où chacun aurait la charge de se guérir «[lui]-mesme». L'adresse au médecin qu'exhibe la lettre publiée s'en trouve justifiée: quand bien même le malade connaît sa complexion et la "vertu" des remèdes ${ }^{8}$, il a besoin de faire appel à un professionnel. Cette exhibition du lien qui l'attache aux médecins se retrouve dans la lettre qui clôt le second tome du recueil (II, 784-793). Il s'agit d'un texte qui prend la forme d'un dialogue entre deux personnages, «Pasquier» et «le Medecin». S’il n’exclut pas la critique, l'échange a pour cadre général, posé au début de la lettre, la guérison de «Pasquier» par «le Medecin». Les arguments polémiques contre la médecine sont ainsi encadrés par une attestation de son utilité, qui justifie que l'on continue à s'adresser aux médecins.

Mais c'est à un niveau plus général, social et politique, qu'il faut concevoir la volonté de Pasquier de conserver un lien avec le corps médical. À la fin de la lettre XIX, 16, Pasquier écrit:

Quoy doncques? me dira quelqu'un, \& paraventure non sans propos, ayant fait tous les discours que dessus. Vous estes d'advis qu'il faut bannir la medecine des Republiques, comme estant chose

7. L'argument des «nations sans médecin» est pris de Pline l'Ancien 1962:23.

8. Dans les traités médicaux de l'époque, la rhubarbe a en effet une «vertu restreignante et [...] expulsive». Sur ce point, voir Brancher 2004. 
indifferente, ou pour mieux dire, un Art, qui en la fragilité de nos sens, est introduit pour nous tromper? (II, 558)

On voit ici que la dévalorisation de la médecine sur le plan épistémique est immédiatement envisagée par Pasquier, sur le plan politique, en termes de «bannissement». Mais, contrairement à Montaigne, qu'il approuve implicitement, l'épistolier ne saurait se résoudre à cette exclusion. À la toute fin de la lettre, on peut lire cette phrase qui explique sa position:

Nous avons la faculté de medecine, l'un des principaux membres des Universitez de nostre France: faculté, dy-ie, fondee sur la doctrine d'Hipocrat, Galien, \& des Arabes, en laquelle outre ce qui est de leurs preceptes, $\&$ receptes, ils ne reiettent pas les Simples qui naissent chez nous, selon que les maladies les requierent. Demeurons sagement en $\&$ au dedans de ceste police; ny ne prenons matiere de nous en plaindre, si quelques particuliers Medecins en abusent: Non plus que ne devons changer nostre Religion ancienne, pour les abus qui se trouvent quelquesfois aux Prestres. (II, 560-561)

Nous reviendrons plus tard sur la question des "Simples qui naissent chez nous", centrale dans le débat que mène Pasquier contre les médecins. Notons pour l'instant que la fidélité à la "faculté de medecine» est fondée sur le parallèle - qu'on devine anxieux pour un homme qui a vécu dans toute leur durée les guerres de religion -, avec la fidélité à la "Religion ancienne», c’est-à-dire catholique. Dans cette mesure, et malgré les réticences que la médecine peut susciter, il faut conserver la "police» traditionnelle. Ce que cherche à conserver Pasquier, ce n'est pas un dogme - puisque, de toute façon, la médecine n'en a pas -, mais une institution. D'où la question centrale de cette lettre: comment conserver une institution sans dogme autour duquel l'organiser?

\section{Les deux «diversifications» et l'institution humaine de la médecine}

Pour maintenir une institution médicale sans dogme, Pasquier va mettre en avant l'efficacité pratique ou thérapeutique de cet art, plutôt que son bien-fondé théorique. Dans notre lettre, le passage d'une impertinence dogmatique de la médecine à son efficacité pratique se fait autour d'une notion centrale: celle de «diversification».

Comme l'annonce Pasquier dans son introduction, la lettre s'organise selon trois parties: la première, que nous avons déjà évoquée, traite du "principal subiect de cest art", c'est-à-dire du corps humain; la seconde, de la discipline médicale elle-même, en deux sous-parties, "la theorique, \& en apres la pratique»; enfin, la troisième expose "sans dissimulation, [le] iugement [de l'auteur] sur cette matiere» (II, 538). Du point de vue de la notion qui nous intéresse ici, celle de "diversification», la première partie sur le «sujet» et la première sous-partie sur la «theorique » de la discipline médicale ont la même valeur argumentative: les deux tendent à montrer qu'il n'y a "rien si certain, que l'incertain en cest art», et en déduisent l'impuissance de la médecine. Comme nous l'avons vu, la première partie prend comme exemple deux éléments définitoires du corps humain controversés parmi les médecins - la question de la priorité des membres ou du cœur dans la conception du corps, et celle de la composition du cerveau -, pour conclure à l'impossibilité de trancher ces débats et, partant, à l'inefficacité de la médecine pour nous soigner. La première sous-partie du second volet, qui traite de la "théorique» de la discipline médicale, s'ouvre sur le constat d'une impuissance de l'esprit humain à atteindre une vérité en médecine: 


\begin{abstract}
Nature plus indulgente envers les autres animaux, qu'envers nous, leur envoyant des maladies qui leur sont diversement propres en leurs especes, les fit aussi Medecins d'eux mesmes, par un certain instinct de leurs natures: \& nous laissa pour nostre partage l'intellect, (amusoir de nostre sotte ambition) sur lequel chacun bastit ses particulieres raisons, que ie ne diray point bastardes, mais bien les appelleray-ie opinions. Voyez doncques en combien de façons sur ce pied s'est diversifié l'art de la Medecine, sur lequel nous establissons les remedes de nos maladies. (II, 544-545, nous soulignons)
\end{abstract}

Tout se joue ici autour du polyptote sur le radical «diver-»: il y a, pour le dire simplement, une bonne et une mauvaise "diversification", en ce sens que la première est au principe d'une efficacité thérapeutique, tandis que la seconde entraîne l'impuissance de la médecine. La bonne est celle des animaux: chaque espèce d'animal a ses propres maladies, mais possède aussi, par "instinct", ses propres remèdes. Au contraire, chez l'homme, qui possède "l'intellect», la diversification des "opinions" médicales éloigne de toute vérité stable, ce qui le condamne à l'inefficacité thérapeutique. Reprenant l'argumentation de Pline, Pasquier se lance alors dans une histoire de la médecine qui retrace les étapes de cette diversification: la doctrine d'Hippocrate est «terrass [ée]» par celle de Chrysippe, lui-même contesté par Asclépiade, lui-même «renvers [é] » par Antonius Musa; arrive alors Thessale, qui «intervertit tout l'ordre ancien de la medecine», auquel succède Galien qui le conteste, et enfin, Paracelse, contemporain de Pasquier, qui "produisit une medecine du tout contraire en principes, à celle d'Hippocrat $\&$ Galien» (II, 545-546). Toutes ces révolutions, dans la mesure où elles relèvent d'une mauvaise diversification, ont pour conséquence l'inefficacité thérapeutique des remèdes humains.

Dans la suite de son développement, Pasquier s'émancipe pourtant du modèle plinien en retournant la valeur de la diversification dans la médecine humaine. Celle-ci, néfaste dans la "Théorique», devient positive dans la "Pratique». La première sous-partie aborde la question des remèdes (II, 549551). À ce sujet, contre les remèdes des médecins de son temps «qui gis[ent] ordinairement en Potions, ou pillules, dont nous allons rechercher les principales estoffes au Levant, \& autres païs estrangers ", il propose de revenir à un principe hippocratique:

Chaque païs a son air \& temperature, de laquelle nous empruntons diversement les habitudes de nos corps \& de nos esprits. [...] Et vrayement la nature auroit esté grandement marastre, \& ingrate, si envoyant les maladies en chaque contree selon la disposition de l'air, elle n'y avoit aussi produit les simples, herbes, arbres $\&$ autres moyens pour les guerir. (II, 549-550, nous soulignons)

De là, il déduit que l'importation de remèdes étrangers est inutile et que les médecins doivent utiliser des remèdes locaux. Le raisonnement reprend en fait, dans sa structure, la bonne diversité des animaux «Medecins d'eux-mesmes». Il suffit de remplacer la notion d' "espece» par celle de «lieu»: la nature, qui fait bien les choses, diversifie les maux par "especes» ou par "lieux», mais donne aussi accès aux remèdes de ces maux, par l' «instinct» ou par la recherche des «simples» locaux. Dans cette mesure, la médecine humaine, tout comme la médecine animale, a tout intérêt à diversifier ses remèdes, au lieu de vouloir uniment s'en procurer du «Levant».

Ce bénéfice de la diversification pour la médecine humaine est confirmé lorsque Pasquier plaide pour une consultation longue du patient par son médecin (II, 552-555). Ce plaidoyer a pour principe une définition de l'homme, c'est-à-dire du "sujet» de la médecine, comme une entité qui varie en fonction de 
[1] a varieté des saisons, des lieux, des aages: \& encores dedans ces aages, la difference de nos mœurs, des nourritures, \& semblablement de la force, estans les aucuns de leur nature plus rares \& flouëts, les autres plus robustes. La diversité qu'il y a aux humeurs qui diversement sont logees en uns \& autres, les uns pour estre sanguins ou coleriques plus faciles à esmouvoir, \& les autres plus difficiles, pour estre possedez par une melancholie sombre \& noire. (II, 552-553, nous soulignons)

Comme dans la première partie de la lettre, le «sujet» de la médecine est à nouveau présenté comme infiniment variable; cependant, ce n'est plus pour conclure à l'impuissance de la discipline, mais au contraire pour indexer son efficacité à sa capacité d'adaptation à des complexions individuelles. Par ailleurs, ce n'est plus, comme dans la citation précédente, le lieu qu'il convient de prendre en compte prioritairement, mais l'individu. Le lieu n'est plus qu'un des critères en fonction desquels le médecin doit déterminer, dans l'exercice de la consultation, le remède adéquat. On constate que Pasquier, d'une certaine manière, radicalise son plaidoyer en faveur d'une "diversification» de la médecine humaine: d'abord, en prenant en compte le lieu, ensuite en s'intéressant à chaque individu.

La diversité n'est donc pas seulement ce qui détermine une impuissance de la médecine, mais au contraire ce qui décide de son efficacité thérapeutique. Ainsi, les médecins peuvent avoir une utilité sociale - guérir effectivement, sans s'adonner de manière trop abstraite à la théorie - dans la mesure où ils respectent cet impératif de diversité. Cette redéfinition de la diversité en médecine permet à Pasquier d'envisager un modèle institutionnel qui assure une intégration sociale à cette discipline, enfin expurgée de ses prétentions théoriques:

On dict qu'anciennement au Temple d'Esculape on affichoit toutes les receptes \& observations pratiquees pour les guerisons, dont Hipocrat composa une partie de ses œuvres. Si nous faisions le semblable, \& que quelque brave compilateur se donnast le loisir de mandier des nostres les remedes qui naissent dedans nostre France contre les maladies, $\&$ de la pluralité d'iceux, fit un choix par un sage iugement $\&$ concert avec autres experts, croyez que nous n’aurions de là en avant grand besoin de caïmander des drogues au Levant, dont nous façonnons auiourd'huy nostre medecine. (II, 551)

Une telle institution, copiée sur le «Temple d'Esculape», permettrait de prendre en compte la "pluralité» des remèdes autochtones et d'enregistrer ainsi, grâce aux «compilateurs », les variations locales de la thérapeutique. Mais elle donnerait aussi la possibilité de réduire cette "pluralité» par un effort commun de synthèse, de manière à fonder une thérapeutique nationale. C'est cet objectif qui assure au corps des médecins, dont le rôle est ici redéfini, une intégration au corps politique du Royaume. Cette volonté d'intégration est par ailleurs lisible dans la réécriture que ce texte impose au modèle plinien. Pline faisait intervenir Hippocrate dans son histoire de la médecine du début du livre XXIX, où il accuse celle-ci de s'être substituée aux remèdes que l'on peut trouver dans la nature: le médecin grec aurait fait brûler le temple d'Esculape où le peuple notait ses recettes, non sans les avoir préalablement recopiées. Ainsi il créa la science nommée "clinique». Pasquier ne mentionne pas la destruction du temple et la responsabilité d'Hippocrate. Le rôle du médecin est alors tout à fait modifié: chez Pline, il confisque les remèdes naturels au peuple; chez Pasquier, il s'inspire des remèdes populaires pour mettre sur pied sa propre médecine. Savoir populaire et savoir médical ne sont plus désormais concurrents, mais complémentaires. Et le personnage d'Hippocrate devient une figure "alternative» au médecin "théoricien": celle d'un "compilateur» qui recueillerait les remèdes populaires et les trierait "par un sage iugement \& concert avec autres experts». Au sévère réquisitoire que Pline et son modèle Caton prononcent contre la médecine, et à l'autarcie thérapeutique qui en découle, Pasquier substitue une critique qui n'exclut pas la collaboration avec les savoirs et les institutions médicales qui en sont la cible. 
De théoricien qu’il était, le médecin est donc passé au statut de «brave compilateur», ce qui modifie tout à fait la manière de concevoir l'institution médicale. Celle-ci ne s'organise plus autour d'un dogme formulé par un savant dont la position est centrale, mais, beaucoup plus horizontalement, par l'agrégation de savoirs dispersés permise par le «concert» des «compilateurs». On songe à l'opposition qu'ont formulée Steven Shapin et Simon Schaffer entre les principes d'organisation scientifique proposés par Hobbes et par Boyle. Alors que le premier pose que «l'activité du philosophe ne connaît pas de limites», et que "l'intelligence est maîtresse incontestée des yeux et des mains", c'est-à-dire des savoirs pratiques, le second propose une "organisation expérimentale», c'est-à-dire une "communauté organique dans laquelle chaque élément dépend inéluctablement de tous les autres, une communauté qui rejette une hiérarchie rigoureuse contrôlée par un maître ". Cependant, si cette "communauté expérimentale» ne doit pas être une "tyrannie», elle n'est pas non plus une "démocratie", au sens où elle donnerait la voix au peuple (Shapin et Schaffer 1993: 336-339). De la même manière, chez Pasquier, le «peuple» n'est pas directement invité au «concert» des "experts». Les «compilateurs» assurent une mission de représentation du peuple qui est autant une tâche de transmission de ses savoirs que de séparation d'avec lui. Reste que, chez Pasquier, contrairement à la description que donnent Shapin et Schaeffer de la situation anglaise, l'autorité du savoir établie par le recueil des " compilateurs» est moins fondée sur la notion d' «expérience» que sur le fait qu'ils réunissent en un lieu unique des remèdes enregistrés dans des endroits divers. Cette assemblée ni tyrannique ni démocratique fonde un savoir pertinent parce qu'il est local (et non parce qu'il est expérimental).

Malgré la fécondité d'une telle proposition institutionnelle, on n'en trouvera pas de prolongements dans la lettre qui nous intéresse. En revanche, l'unité de la médecine divisée y est assurée, par un autre moyen: la présence de Dieu, garant d'une vérité de la discipline et dont les médecins sont de simples agents.

\section{Les «contrariétés» des dogmes médicaux et l'institution divine de la médecine}

Ce modèle divin apparaît au début de la troisième partie. L'épistolier introduit son propos par l'intervention d'un interlocuteur imaginaire, qui tirerait une conclusion plinienne du constat épistémique posé par Pasquier, celui d'un dogme médical divisé:

Quoy doncques? me dira quelqu'un, \& paraventure non sans propos, ayant fait tous les discours que dessus. Vous estes d'advis qu'il faut bannir la medecine des Republiques, comme estant chose indifferente, ou pour mieux dire, un Art, qui en la fragilité de nos sens, est introduit pour nous tromper? Ia à Dieu ne plaise, que telle soit mon opinion: Mais au contraire, c'est un art, par lequel sur tous les autres ie cognois les miraculeux effects de la puissance de Dieu nostre souverain Medecin. Car combien que tous les grands Medecins, dont ie vous ay cy dessus parlé, fussent differents en leurs principes, remedes, \& conduite de leur profession; toutesfois en ceste contrarieté, Dieu voulut qu'ils guerissent une infinité de malades, qui eurent vers eux recours, voire en grandes maladies, que l'on estimoit hors d'espoir. (II, 558)

Pasquier revalorise ainsi la profession médicale par l'effectivité de la guérison, qu'il attribue à Dieu. On note une légère inflexion lexicale, qui nous paraît révélatrice d'une inflexion du sens à l'échelle de la lettre: plutôt que de "diversification", Pasquier parle ici de "contrariété». Or, cette requalification de la division doctrinale en "contrariété» n'est pas indifférente. On l'a vu, la division dogmatique peut 
être envisagée comme une "bonne diversification" dans la mesure où elle prend en compte la variété des lieux et des patients, et assure par là une efficacité thérapeutique. Si l'on décrit en revanche la division des dogmes médicaux comme une "contrariété", on met en valeur le pouvoir de Dieu, notamment sa capacité à réunir les contraires en une unité, à produire un accord parmi les discordances. Dès lors, rien d'étonnant à ce que les actions des médecins qui sont données en exemple de ces "miraculeux effects de la puissance de Dieu» ne soient pas décrites selon les bonnes procédures thérapeutiques de la «diversification» que Pasquier a présentées plus haut. En effet, on apprend qu' "Asclepiade avec ses preceptes nouveaux \& paradoxes redonn[e] la vie à un homme», que Thessale "fit d[']estranges miracles", que Paracelse a "guery quelques hommes de leur lepre, maladie notoirement incurable" (II, 559) : mais à aucun moment on ne sait s'ils ont appliqué les préceptes de la bonne thérapeutique que l'épistolier vient pourtant d'exposer. Dieu semble guérir indépendamment d'une bonne ou d'une mauvaise pratique thérapeutique; peu lui importe la «diversité» des patients, puisqu'il a la puissance absolue de redonner la santé par le truchement de théories médicales contraires.

Mais ce modèle divin de l'institution de la médecine ne s'énonce pas du tout de la même manière que le précédent. Là où le modèle humain ne se disait qu'au détour de l'évocation d'une institution antique et n'était jamais développé, le modèle divin, déjà explicité dans le précédent passage, est à nouveau affirmé dans les deux dernières pages de la lettre, comme pour exempter l'auteur d'une possible accusation de commettre un texte contre la Faculté de Médecine:

En ceste contrarieté de preceptes, à qui doit on les guerisons? A ce grand \& souverain Medecin nostre Dieu, lequel pour la conservation du genre humain, voulut $\&$ veut; que par l'entremise des Medecins, la santé nous fust renduë. (II, 560)

[E]n l'exercice de cest art on y voit les miracles tres expres de Dieu [...]. (II, 561)

Par ailleurs, au-delà de cette fonction d'encadrement idéologique d'un propos qui pourrait être mal interprété, on trouve ce modèle divin, plus discrètement, au principe d'une manière particulière d'écrire les anecdotes dans cette lettre. Cela est particulièrement clair au moment où Pasquier décrit les divisions médicales par le biais d'une brève histoire des dissidences dogmatiques, et où il reprend cette histoire pour rappeler que ces dissidences, malgré leur contrariété, guérissent. Dans la première série d'anecdotes, il s'agit donc d'exposer une impuissance de la médecine à force de division. Reprenant Pline, Pasquier conclut ainsi :

Tout cela n'est ce pas donner des dementirs les uns aux autres, non aux despens de leurs vies (comme font ceux qui combatent en camp clos) ains aux despens des nostres, qui ne pouvons mais de leurs querelles? (II, 546)

La division de la médecine et les "dementirs» que les différents dogmes médicaux s'opposent les uns aux autres sont responsables de la mort des patients. Comme chez Pline, le malade «meurt de trop de médecins", mais, telle que la raconte Pasquier, cette conclusion paraît presque inappropriée. Voici par exemple comment est décrite l'action de Chrysippe, successeur d'Hippocrate:

encores qu'il [Hippocrate] fust $\&$ le premier, $\&$ le plus singulier en cest art, si eut-il quelque temps apres un Chrysippe successeur, qui par une malice affectee voulut terrasser sa doctrine, \& neantmoins en ceste cacochimie d'esprit, eut tres-grande vogue parmy les siens, \& fut en reputation du plus grand Medecin de son temps pour les cures admirables qu'il faisoit. (II, 545) 
Puis celle d'Antonius Musa:

Et apres luy [Asclépiade] du temps de l'Empereur Auguste, un Antonius Musa, qui fit le semblable, $\&$ renversa toute la doctrine d'Asclepiade, en cecy favorisé de son Maistre, qu'il avoit guery d'une maladie que l'on estimoit incurable. (II, 545)

Ces deux anecdotes se signalent par une valeur argumentative ambiguë et identique. Considérées dans le cadre de la satire, elles peuvent être interprétées ainsi : chaque médecin prétend détruire («terrasser», «renverser») la doctrine précédente, et propose une nouvelle doctrine qui est indument valorisée par la société («les siens» pour Chrysippe, Auguste pour Musa). Mais, par rapport à la conclusion inspirée de Pline (ces querelles se font aux dépens de nos vies), il y a un hiatus : c’est que ces nouveautés médicales guérissent bel et bien. Or, c'est bien cette effectivité de la guérison qui va permettre par la suite à Pasquier de sauver les médecins: derrière ces querelles, il y a Dieu, "nostre souverain Medecin ", qui envoie ceux-ci pour produire une infinité de guérisons. Ainsi, la présence d'un Dieu caché, qui guérit malgré l'évidence d'un dogme médical divisé par les querelles, est dissimulée à ce stade de l'argumentation, mais elle se laisse deviner derrière le hiatus existant entre l'idée explicitée par Pasquier (l'impuissance de la médecine divisée) et les exemples (qui montrent la persistance de certaines guérisons).

Or, lorsque Pasquier reprend cette histoire pour la corriger et mettre en avant cette fois-ci la capacité de la médecine à guérir, il reprend ce principe d'anecdotes dont la valeur argumentative ambiguë dissimule une intervention divine:

Ainsi raconte l'on qu'Asclepiade avec ses preceptes nouveaux \& paradoxes redonna la vie à un homme, duquel (comme mort) on estoit sur le point de faire les funerailles, en luy faisant boire du vin accommodé à sa guise. Et fit mesmement une protestation bravasche, qu'il ne vouloit estre tenu pour Medecin, si on le voyoit iamais malade, ou mourir d'une maladie. Et luy advint ainsi qu'il avoit protesté, estant arrivé à une extreme vieillesse sain \& sauf, iusques à ce que monté sur une eschele, l'un des eschelons se rompit sous ses pieds, qui le fit trebuscher du haut en bas dont il mourut sur le champ. (II, 559)

En plus d'être ici l'instrument d'une mort «inopinée» (comme l'indique la manchette en marge de l'anecdote), l'échelle est sans doute le symbole de la chute d'un médecin qui, par une "protestation bravache", a voulu se substituer à Dieu, et qui s'est mis à croire à sa propre efficacité thérapeutique au point d'oublier l'origine divine de la guérison. Cette hypothèse est confirmée par le second éloge, lui aussi ambigu :

Le Thessalus qui sous l'Empire de Neron renversa avec bravade la doctrine de ses predecesseurs, fit de si estranges miracles, en l'exercice de sa medecine, que apres sa mort on mit sur son tombeau ces deux mots, Qu'en ce lieu gisoient les os de Thessale, en son vivant le Prince des Medecins. (II, 559)

Le travail de Pasquier par rapport à sa source est ici particulièrement habile. Chez Pline, l'orgueil de Thessale se manifeste par le fait qu'il se proclame lui-même, dans son épitaphe, prince des médecins. Ce trait complète donc la satire plinienne de la profession médicale, coupable de s'émanciper indument du savoir-faire populaire. En ne mentionnant pas que Thessale s'est attribué à lui-même le titre de prince des médecins, Pasquier atténue la critique plinienne: c'est bien "on» (le peuple, éventuellement) qui, grâce aux guérisons qu'il a effectuées, attribue à Thessale le titre de «Prince de Medecins». Cependant, en évoquant l'idée de «bravade», qui rappelle la "protestation bravache» d'Asclépiade, et 
en juxtaposant, dans l'épitaphe, la mention des «os de Thessale» avec l'expression "en son vivant», Pasquier introduit une autre nuance à ce tableau: celle d'un memento mori. Ce qui est implicitement reproché alors à Thessale, ce n'est plus d'avoir usurpé la réputation de bon médecin, mais d'avoir oublié sa condition de mortel; d'avoir oublié, "de son vivant», qu'il ne serait bientôt plus que des "os». Même s'il guérit, le médecin est condamné à mourir et ne peut prétendre à une condition divine et immortelle. Ainsi, les deux anecdotes, qui tendent à nous montrer la capacité de la médecine à guérir, nous prouvent aussi, par le hiatus entre l'idée générale qu'elles doivent illustrer et ce qu'elles racontent effectivement, le pouvoir de Dieu de rappeler à ses agents leur qualité de subordonnés, lorsque leur orgueil fait croire à ces derniers qu'ils égalent le Créateur.

Dans ces deux séries d'anecdotes (celles qui prouvent l'impuissance de la médecine, et celles qui prouvent sa capacité à guérir), le lieu textuel de la puissance de Dieu est toujours dans l'implicite de l'anecdote. On peut donc en déduire que les célébrations explicites de la puissance de Dieu, qui se multiplient, on l'a vu, à la fin de la lettre XIX, 16, ne sont pas de pures formes, au sens où elles n'encadrent pas un texte qui n'en parlerait pas, voire qui dirait l'inverse. Au contraire: l'explicite et l'implicite du texte se rejoignent dans une même célébration de la puissance de Dieu. Cependant, on ne peut ignorer non plus ce qui semble échapper à la cohérence d'une médecine d'origine divine qui rassemblerait sous elle la "contrariété» des diverses écoles médicales - à savoir le modèle d'une médecine qui, prenant en compte la diversité des lieux et des personnes, se proposerait d'enregistrer les variations thérapeutiques et de formuler, moyennant un tri par des "sages», une thérapeutique nationale. Tout le problème de la lettre réside dans l'articulation de ces deux modèles qui, à aucun moment, n’est explicitée.

\section{Penser l'articulation des deux modèles}

Une première réponse à cette question serait d'affirmer que la distinction entre institution divine et institution humaine de la médecine, que nous avons posée, est anachronique; qu'il y a, dans la pensée de l'époque de Pasquier, une continuité entre la pratique médicale au fondement de l'institution humaine de la médecine - la prise en compte de la diversité des patients - et la croyance dans une intervention divine qui permet aux médecins de les guérir. On trouvera dans un article de Josiane Rieu, intitulé «Les résonances ignatiennes de la "pure indifférence” chez Montaigne» (Rieu 2016), des éléments abondant dans ce sens. L'auteure y développe la thèse selon laquelle, dans le contexte de la Contre-Réforme, il n'existe pas de différence entre la recherche de la vérité dans le domaine sensible, et l'idée que, grâce à notre croyance en Dieu, celui-ci nous délivre sa Grâce:

Par opposition à l'idée d'une corruption radicale de la nature humaine, sur laquelle insistait le calvinisme par exemple, la Contre-Réforme (et le Concile de Trente) fait acte [de] confiance dans la Création, et dans la capacité de l'homme à expérimenter, dans la réalité et dans sa propre nature même, la Vérité. (Rieu 2016: n. p., \$16)

Mais cette valorisation du sensible ne va pas, évidemment, jusqu'à l'idée que l'homme peut trouver la vérité dans l'expérience seule. La recherche de la vérité par les moyens proprement humains est possible, dans la mesure où l'homme ne se "ferme» pas et n'utilise pas «ses facultés "pour lui-même", en système clos». En revanche,

si l'homme reconnaît que toutes ses facultés corporelles, spirituelles et intellectuelles sont ordonnées à la vie en Dieu, celles-ci sont totalement réhabilitées, enfin révélées à leur essence: il passe de la 
nature à la surnature, dans ce mouvement d'amour humain qui appelle indéfectiblement l'amour de Dieu et le don de sa Grâce. (Rieu 2016: n. p., \$7)

L'effort de l'esprit humain vers la vérité peut ainsi être traduit comme une "phas[e] de purification (des sens, du jugement, de l'affection...), en vue de parvenir à la croissance de l'âme et à la pleine union à Dieu» (Rieu 2016: n. p., \$8). Josiane Rieu analyse cette idée chez Ignace de Loyola, pour conclure que «[l]a prise en compte de la réalité sensible de l'homme dans la spiritualité ignatienne est relayée par Montaigne» (Rieu 2016: n. p., \$18) : ainsi, cette importance du sensible chez Montaigne, notamment dans sa méthode de connaissance, où l'on a pu voir le signe d'un épicurisme de l'auteur, ne serait en fait qu'une trace de cette revalorisation post-tridentine de l'expérience proprement humaine.

Ces considérations, qui rétablissent un lien ferme entre Montaigne et la religion catholique ${ }^{9}$, pourraient être importantes pour notre lecture de la lettre de Pasquier sur la médecine. En effet, l'opposition entre un modèle médical divin et un modèle médical humain n'est peut-être qu'une vue d'un esprit moderne; dans ce modèle post-tridentin, une continuité semble pouvoir être établie entre la recherche humaine d'une efficacité thérapeutique par la prise en compte de la diversité des lieux et des individus d'une part, et l'intervention divine qui permet la guérison d'autre part. C'est pourquoi Pasquier ne s'attarderait pas sur ce modèle humain d'institution médicale, qui ferait des médecins des "compilateurs»; ce que nous avons aperçu comme un modèle médical humain ne serait en fait qu'une préparation à recevoir l'illumination de la Grâce divine. Sans doute faudrait-il examiner plus en détail les conceptions théologiques de Pasquier, mais, si l'on s'en tient à cette lettre, il semble que cette hypothèse d'une continuité ne tienne pas. D’abord, comme nous l'avons déjà noté, Pasquier ne précise pas selon quels préceptes médicaux les médecins «envoyés de Dieu» agissent. Peu importe, semble-t-il, l’utilisation qu'ils font de la raison: Dieu intervient par la guérison, dans une indifférence totale à leurs diverses pratiques médicales. Par ailleurs, rien n'indique dans cette lettre que la recherche de la vérité par des moyens purement humains soit considérée comme une "purification» ou une étape vers la possibilité d'être frappé par la Grâce divine. On note au contraire une sorte de hiatus non explicite, à l'intérieur même de la pensée de Pasquier, entre une médecine hasardeuse, qui se révèle finalement être une médecine divine (ce qui est perçu comme hasard par l'homme étant en réalité une intervention de Dieu), et une médecine humaine, qui procède par localisation et individualisation des patients, puis attribution du remède adéquat. Si continuité il y a entre ces deux modèles, elle n'est pas lisible dans cette lettre.

C'est pourquoi nous voudrions ici envisager une autre manière de penser leur articulation. Pour ce faire, il faut aborder un problème central de cette lettre: celui de la diffusion des discours sur la médecine en langue vernaculaire à la fin du Xvi ${ }^{\mathrm{e}}$ siècle.

En écrivant une lettre sur la médecine en langue vernaculaire, Pasquier participe non seulement à la progression de la langue française dans le genre épistolaire ${ }^{10}$, mais contribue aussi au large mouvement de vernacularisation du discours médical, entamé depuis les années $1530^{11}$. Celui-ci répond à un impératif pratique de formation d'élèves de moins en moins versés dans la langue latine, mais aussi à une volonté d'indépendance par rapport à «l'étranger» qui rejoint très exactement les ambitions de constitution d'une thérapeutique nationale que l'on observe chez l'épistolier. Magdalena Kozluk cite ainsi le sonnet panégyrique qu'en 1596 Abraham de Saint Loup adresse à Honoré l'Amy, auteur

9. Francis Goyet cite longuement l'article de Rieu dans un chapitre de ses Audaces de la Prudence éloquemment intitulé "Que Montaigne est profondément catholique» (Goyet 2009: 97-108).

10. Sur ce point, voir Catherine Magnien 1999: 6-83 et 115-156, ainsi que Vaillancourt 2003: 136-146.

11. Sur ce point, voir Carlino 2009. 
d'un Abbregé chirurgical. Il le loue d'avoir fait une compilation en français à partir de textes médicaux grecs, et se félicite de ce que, grâce à ce "larcin», "[l]e François n'ira plus mendier hors de France,/ [1] es secrets pour donner au malade allegeance» (L'Amy 1596: fo A 7 r ${ }^{\circ}$; cité dans Kozluk 2012: 85). La formulation est très similaire à celle qu'utilise Pasquier lorsqu' esquissant le projet d'une institution médicale nationale dans laquelle des médecins «compilateurs" recueilleraient les remèdes locaux, il se réjouit que l'on n'ait plus "de là en avant grand besoin de caïmander des drogues au Levant». Le projet de constitution d'une thérapeutique nationale semble ainsi regrouper l'ambition d'une compilation des remèdes français et celle d'une vernacularisation du discours médical. En effet, pour qu'une collaboration soit possible entre médecine savante et médecine populaire et que le "compilateur" puisse recueillir les remèdes locaux, il faut une langue commune: Hippocrate n'aurait pas pu relever les remèdes dans le temple d'Esculape s'il n'existait pas une langue médicale grecque commune à la fois au peuple et aux savants.

Cependant, même chez les partisans de la vernacularisation, la question de la diffusion du savoir médical soulève des difficultés. Ainsi, le grand médecin montpelliérain Laurent Joubert, proposant en 1579 une nouvelle traduction en français de la Grande Chirurgie de M. Gui de Chauliac, justifie son travail par le manque de clarté de la précédente traduction:

[...] la traduction de Latin en François estoit si lourde, scabreuse, grossiere, barbare \& espineuse, qu'il n'y avoit moyen de la corriger: il l'ha fallu toute refondre, \& faire de nouveau. Vrayement il y ha plusieurs endroicts si broulhés \& tenebreux, qu’ils semblent des ænigmes. (Joubert 1579: $5^{12}$ )

Cette volonté de clarté, qu'accompagne le souci d'une meilleure diffusion de la Chirurgie de Chauliac, va ainsi de pair avec la vernacularisation du savoir chirurgical. Pourtant, cette diffusion a ses limites, du moins dans l'idée a priori qu'en a Joubert, puisqu'elle n'inclut pas les lecteurs «ignorants»:

Vray est que les ignorants de cest art, la [la langue de la traduction de la Chirurgie] trouveront asses difficile, parce que i'ay voulu retenir la gravité du stile medicinal, les termes, phrases \& sentences usitees, en nos escoles: comme en tous arts $\&$ sciences il y ha autre langage, que le commun $\&$ familier, bien que le tout soit en vulgaire, en Latin, ou en Grec. Aussi faut il que les bons livres, qui traictent des matieres plus serieuses, comme font ceux qui contiennent les loix de Dieu, de Nature, $\&$ des Hommes, pour nous faire vivre religieusement, sainement, \& paisiblement, a la conduicte des Theologiens Medecins \& Magistrats, soyent tellement publiez en quelque langue que ce soit, qu'on puisse dire comme Aristote, respondant au grand Alexandre de ses acroamatiques, Ils sont divulguez $\&$ non divulguez. Car il fault tousiours neantmoings aller a l'escole, $\&$ ouyr là dessus ceus qui ensegnent d'un oracle de vive voix. (Joubert 1579: 5-6)

L'enjeu du maintien, malgré la traduction française, de la "gravité du stile medicinal», est social: il en va de l'autorité des "Théologiens Medecins \& Magistrats", chargés de nous faire vivre "religieusement, sainement, \& paisiblement». Le style "grave», qui est utilisé «en nos escoles», permet de trier les publics touchés par cet écrit. Seuls ceux qui sont allés à l'école pourront le comprendre. Donc, malgré

12. Je remercie Sylvie Bazin-Tacchella d'avoir attiré mon attention sur l'enjeu de la vernacularisation du discours médical dans cette œuvre, lors de sa communication «Défense et illustration d'une chirurgie savante en français: l'édition et la traduction de La grande chirurgie de Gui de Chauliac par Laurent Joubert», à l'occasion du colloque " En toutes sortes communiquer le plus secret de sa science" : Laurent Joubert, médecin et écrivain (1529-1582)» (Lyon, 21-23 juin 2018), organisé par Ariane Bayle, Dominique Brancher et Michel Jourde. 
la diffusion incontrôlable de l'imprimé, la langue permettra de ne transmettre ces savoirs, si précieux pour la cohésion sociale, qu'à ceux qui ont été institutionnellement formés pour les manier.

Le problème de la diffusion du discours médical, à laquelle Pasquier travaille en écrivant sur la médecine en langue française et en formulant le projet d'une collaboration entre médecine savante et médecine populaire, est abordé par l'épistolier dans des termes assez similaires à ceux de Joubert. Dans la dernière lettre du recueil, présentant, comme on l'a vu, «un dialogue » entre deux personnages, «Pasquier» et «le Medecin», le second réagit ainsi aux vives critiques du premier:

[...] vous oyant en ce point parler des Medecins, il sembleroit que pendant vostre maladie eussiez conceu quelques fausses imaginations contr'eux: sur lesquelles vous seriez ferme revenu en vostre santé. Et neantmoins vous sçavez que ce que dites ne se peut soustenir avec fondement de raison: voire que vostre opinion pourroit se tourner en consequence, estant communiquee à un peuple. (II, 785)

Là encore, le problème d'une critique de la médecine formulée en langue française est celui de sa "communi[cation] à un peuple», c'est-à-dire un problème d'autorité de la catégorie sociale qui est, normalement, en charge de la discipline. Et cette mise en garde, ici attribuée au "Medecin ", se retrouve dans la lettre XIX, 16, assumée cette fois-ci par Pasquier lui-même. À la fin de la partie qui concerne la "pratique» de l'art médical, il écrit cette condamnation sévère à l'égard de la médecine telle qu'elle est exercée de son temps:

[...] si i'ozois, ie dirois voluntiers, que la guarison qu'en [de la médecine] raportons procede plus du hazard que de l'art, avec l'aide de la force de nostre nature, à laquelle nous rendons la principale grace à l'issue de nos grandes maladies, comme si on vouloit dire, que la medecine est seulement introduite pour tromper les bources des gens riches $\&$ aisez qui veulent estre trompez. Pour le moins un pitaut de village ne doutera de le dire, lequel affligé d'une fievre tierce, en sera garenty au septieme accés sans rien prendre, aussi bien que le Citoyen \& Bourgeois, lequel voulant par aposumes, clysteres, medicaments \& saignees forcer par impatience la nature du mal, a peine avec toutes ces flateries fascheuses, qu'il ne passe par autant d'accés que l'autre. (II, 555)

«Si i'ozois»: la prétérition, bien évidemment, n’empêche pas la formulation de la critique. De fait, Pasquier participe à la diffusion de l'idée que la médecine est impuissante. Mais, en même temps, il formule la crainte que cette critique se diffuse dans les classes inférieures de la société. L'argument semble légèrement différent de celui du "Medecin", dans la mesure où, ce qui risque de propager la critique de la médecine, c'est moins le texte de Pasquier que le peu de résultat de la discipline en elle-même, que constate le "pitaut de village» lorsqu'il compare son état de santé à celui du "Citoyen \& Bourgeois» qui use et abuse des thérapeutiques les plus sophistiquées. Pourtant, en soulignant l'aspect lucratif et dissimulateur de l'art médical («tromper les bources»), ce "pitaut de village» parle ici comme Pline. La question de la diffusion de la critique de la médecine mêle ainsi, inextricablement, celle d'une vernacularisation de la critique, et celle du constat empirique de l'impuissance de l'art médical.

On trouve donc, dans la prose même de Pasquier, une forme de paradoxe énonciatif qui rejoint les tensions propres à la vernacularisation de la discipline médicale au tournant des $\mathrm{XVI}^{\mathrm{e}}$ et $\mathrm{XVII}^{\mathrm{e}} \operatorname{siècles.~}$ D'un côté, en proposant un discours médical en français et en promouvant une institution qui favoriserait la collaboration entre médecine populaire et médecine savante, Pasquier se place du côté des vulgarisateurs qui envisagent la création d'une thérapeutique nationale, dans un souci d'indépendance 
par rapport à l'étranger. Mais, d'un autre côté, il semble important pour lui de souligner les limites d'une telle vulgarisation, de manière à ce que l'autorité de l'institution médicale telle qu'elle existe soit respectée. Il nous semble que la cohabitation des deux modèles que nous avons signalés - le modèle divin et le modèle humain de l'institution de la médecine -, doit se comprendre en lien avec ce paradoxe qui traverse la lette XIX, 16 (et l'ensemble du discours médical dans les Lettres). Là où l'institution humaine de la médecine, par la diffusion des savoirs médicaux qu'elle permet, risque de renverser l'ordre préétabli, l'institution divine remet chacun à sa place: le "peuple» en-dessous des médecins, puisque ceux-ci sont des agents divins; les médecins en dessous de Dieu, puisqu'ils ne sont que les agents de Sa puissance. Il faut donc lire, nous semble-t-il, les deux modèles non pas dans une relation de continuité, mais dans une relation de compensation, rendue nécessaire par une tension interne au projet même de Pasquier. Dans la mesure où le modèle humain, pourtant cohérent avec les propositions épistémiques de Pasquier, est trop audacieux socialement - car il risque de diffuser des savoirs médicaux qui, in fine, pourraient se retourner contre l'autorité de la médecine elle-même -, il faut qu'il soit compensé par un autre modèle, divin celui-ci, qui consolide la place que chacun doit tenir. Considérer ces deux modèles en continuité, comme on serait tenté de le faire à la lecture de l'article de Rieu, risquerait de nous faire passer à côté de la tension, ou du «trouble ${ }^{13}$ ", qui est au cœur de la lettre: le conflit entre la vernacularisation du savoir médical au service d'une thérapeutique nationale, et la peur que cette diffusion, en affaiblissant l'autorité de l'institution médicale, n'ébranle les hiérarchies traditionnelles du royaume de France ${ }^{14}$.

On voit donc qu'à mieux considérer les liens entre savoirs individuels et savoirs dogmatiques, l'idée que notre lettre constituerait une célébration de la transgression des seconds par les premiers devient très fragile. La remise en cause du savoir dogmatique ne saurait se faire simplement, dans la mesure où elle évoque immédiatement les discussions du dogme religieux à l'origine des guerres civiles. Qu'il y ait donc un apport thérapeutique d'une plus grande attention prêtée aux particularités de l'individu que le médecin doit soigner, cela semble hors de doute. Mais tout l'intérêt de la lettre de Pasquier est de réfléchir à la manière d'institutionnaliser une telle science des particularités, et aux difficultés d'un tel processus. L'institution divine, qui regroupe les différentes thérapeutiques et les différents cas sous la puissance unique d'un médecin suprême, ne saurait, nous semble-t-il, constituer l'unique réponse de Pasquier. Il faut aussi considérer la solution esquissée par l'évocation du «Temple d'Esculape», qui met à contribution le savoir populaire sous le contrôle des médecins plus savants.

13. Nous empruntons la notion à Terence Cave, qui désigne ainsi, dans certains textes de la Renaissance, des zones d' «incertitude épistémologique» ou d' "angoisse ontologique ou axiologique» (Cave 1999: 15). Dans sa contradiction non résolue, cette lettre nous semble précisément agitée par une angoisse épistémique, qui porte sur la possibilité d'une institution humaine de la médecine et d'une thérapeutique nationale.

14. Béatrice Sayhi-Périgot, lorsqu'elle commente la suppression, après la première édition de 1581, d'un long passage du Pourparler de la loy où Pasquier met en avant la faiblesse de la raison humaine, propose cette interprétation: «Peut-être cette suppression révèle-t-elle que vers la fin du siècle, le scepticisme, dans sa conception fidéiste, n'est plus compris, que le courant rationaliste, en donnant au doute sa teinte pyrrhonienne et "lucianique", en a fait une attitude de provocation intellectuelle, mais aussi sociale, que poursuivra au dix-septième siècle le courant libertin. Or, cette attitude est exactement l'inverse de celle que suivra toute sa vie Pasquier, qui tentera toujours d'allier, à une grande liberté de pensée et à une exceptionnelle largeur d'esprit, un profond respect des valeurs établies, respectables parce qu'elles représentent, patinée par le temps, la vérité la plus probable» (Sayhi-Périgot 1995: 460). On peut faire l'hypothèse que la contradiction que nous voyons dans la lettre sur la médecine a la même fonction que la suppression commentée dans ces lignes: celle de corriger une trop grande audace épistémique et sociale par la réaffirmation de la confiance dans les hiérarchies traditionnelles.

Arborescences - Revue d'études françaises 
Mais cette solution ne va pas sans difficulté, ce qui explique sûrement qu'elle ne soit qu'esquissée. La contribution du peuple à une thérapeutique nationale, et la collaboration avec la médecine savante que cette contribution impliquerait, remet en cause l'idée d'une autorité particulière des médecins et de l'institution médicale. C'est autour du "peuple» que la conception de la médecine de Pasquier semble donc la plus hésitante, puisqu'il est à la fois l'entité qui apporte les connaissances thérapeutiques les plus efficaces, et le groupe qui risque le plus, si l'on diffuse trop ouvertement l'idée que la médecine dogmatique est impuissante, de remettre en cause les hiérarchies qui assurent le bon fonctionnement du royaume de France.

L'inscription du discours médical dans les lettres familières en vernaculaire, dont le recueil de Pasquier, aux dires de l'auteur, sont le premier exemplaire en langue française, n'est donc pas seulement synonyme d'individualisation du savoir médical, au sens où le «je», se considérant comme un «cas», adapterait un savoir général à sa complexion personnelle. À trop considérer les lettres familières - et donc le discours médical qui y est tenu - par le prisme de l' «émergence de l'individu», on risque de perdre de vue un enjeu qui, pourrait-on dire, accompagne comme son ombre l'affirmation des singularités: la recherche d'une forme institutionnelle capable de regrouper ces singularités, et l'entreprise, sans doute encore inaboutie et chimérique au tournant des $\mathrm{XVI}^{\mathrm{e}}$ et $\mathrm{XVII}^{\mathrm{e}}$ siècles, de constituer un corps politique malgré la diversité des individus et des opinions ${ }^{15}$.

\section{Références bibliographiques}

Alazard, F., S. Geonget, L. Gerbier et P.-A. Mellet, dir. 2016. Dissensus: Pratiques et représentations de la diversité des opinions (1500-1650). Paris: Honoré Champion.

Brancher, D. 2004. "Ny plus ne moins que la rubarbe qui pousse hors les mauvaises humeurs": la rhubarbe au purgatoire». Dans L'écriture du scepticisme chez Montaigne, sous la direction de M.-L. Demonet et A. Legros. Genève: Droz: 303-320.

Brancher, D. 2007. «Médecins-médecine». Dans Dictionnaire de Michel de Montaigne, sous la direction de Ph. Desan. Paris: Classiques Garnier: 740-744.

Carlino, A. 2002. "Afflizione e scetticismo: Montaigne e la letteratura contra medicos». Medicina nei Secoli. Arte e Scienza 14 (2) : 479-497.

Carlino, A. 2009. «Introduction». Dans Vulgariser la médecine: Du style médical en France et en Italie (XVI et XVII siècles), sous la direction d'A. Carlino et M. Jeanneret. Genève: Droz: 9-31.

Cave, T. 1999. Pré-histoires: Textes troublés au seuil de la modernité. Genève: Droz.

Fumaroli, M. 1978. "Genèse de l'épistolographie classique: rhétorique humaniste de la lettre, de Pétrarque à Juste Lipse». Revue d'histoire littéraire de la France 6: La lettre au XVII siècle: 886-905.

Gimaret, A. 2010. «Maladie et introspection dans les Lettres familières d'Étienne Pasquier». Dans M@gm@ 8 (1) : Écritures de soi en souffrance, sous la direction de O. M. Valastro. URL: http://www.analisiqualitativa.com/magma/0801/articolo_02.htm_Consulté le 5 septembre 2018.

Gimaret, A. 2011. Extraordinaire et ordinaire des Croix: Les représentations du corps souffrant (1580-1650). Paris: Honoré Champion.

Goyet, F. 2009. Les audaces de la prudence: Littérature et politique aux XVI et XVII siècles. Paris: Classiques Garnier. Joubert, L. 1579. La grande chirurgie de M. Gui de Chauliac..., restituée par M. Laurens Joubert. Lyon: Estienne Michel.

15. Ce dernier point est au cœur du récent collectif publié par le Centre d'études supérieures de la Renaissance: Alazard et al. 2016. 
Kozluk, M. 2012. L'Esculape et son art à la Renaissance: Le discours préfaciel dans les ouvrages français de médecine (1528-1628). Paris: Classiques Garnier.

L'Amy, H. 1596. Abbregé chirurgical. Lyon: Jean Pillehotte.

Magnien, C. 1999. Étienne Pasquier épistolier. Thèse d'habilitation, Université Paris IV.

Montaigne, M. de. 2004. Les essais. Édition préparée par P. Villey. Paris: Presses universitaires de France [1965].

Pasquier, É. 1619. Les lettres, t. I et II. Paris: Laurent Sonnius.

Pline l'Ancien. 1962. Histoire naturelle, livre xxix. Traduit par A. Ernout. Paris: Les Belles Lettres.

Pline l'Ancien. 1972. Histoire naturelle, livre xxıv. Traduit par J. André. Paris: Les Belles Lettres.

Rieu, J. 2016. "Les résonances ignatiennes de la "pure indifférence" chez Montaigne». Loxias 55: Autour des programmes de concours 2017. URL: http://revel.unice.fr/loxias/index.html?id=8564. Consulté le 5 septembre 2018.

Sayhi-Périgot, B. 1995. «Pasquier ou les voix de la vérité. Commentaires des Pourparlers». Dans É. Pasquier, Pourparlers. Édition préparée par B. Sayhi-Périgot. Paris: Champion: 277-544.

Shapin, S. et S. Schaffer. 1993. Leviathan et la pompe à air: Hobbes et Boyle entre science et politique. Paris: La Découverte.

Vaillancourt, L. 2003. La lettre familière au XVI siècle: Rhétorique humaniste de l'épistolaire. Paris: Honoré Champion. 